\title{
Avaliação da toxicidade de diferentes concentrações de tinturas de malva e calêndula através do bioensaio com Artemia salina
}

Evaluation of the toxicity of different concentrations of mallow and marigold tinctures through the bioassay with Artemia salina

Evaluación de la toxicidad de diferentes concentraciones de tinturas de malva y caléndula mediante el bioensayo con Artemia salina

Flávia Gouvêa Costa Tchemra ORCID: https://orcid.org/0000-0002-0752-7409 Faculdade Paulo Picanço, Brasil E-mail: flaviatchemra@gmail.com Mateus Mota Pontes ORCID: https://orcid.org/0000-0002-6249-6094 Faculdade Paulo Picanço, Brasil E-mail: mateus.pontes@facpp.edu.br

Aline Rocha de Melo ORCID: https://orcid.org/0000-0003-1168-8691 Faculdade Paulo Picanço, Brasil E-mail: aline.melo@facpp.edu.br Juliana Larocca de Geus ORCID: https://orcid.org/0000-0001-9633-0474 Faculdade Paulo Picanço, Brasil E-mail: juliana.degeus@ facpp.edu.br

Vitoldo Antonio Kozlowski Junior ORCID: https://orcid.org/0000-0002-1339-4614 Universidade Estadual de Ponta Grossa, Brasil E-mail: vakozlowski@uepg.br

Márcia Rezende

ORCID: https://orcid.org/0000-0001-8474-5656 Faculdade Paulo Picanço, Brasil E-mail: rezendemarcia@outlook.com

\begin{abstract}
Resumo
O objetivo foi avaliar a toxicidade das tinturas de malva 10\% (Malva sylvestris) e calêndula $10 \%$ (Calendula officinalis), através do bioensaio com Artemia salina. Os náuplios foram obtidos e transferidos para tubos de ensaio, os quais continham tinturas de malva e calêndula em diferentes concentrações $(25,50,75,150,250,500,750$ e 1000 $\mu \mathrm{g} / \mathrm{ml}$ ), e substâncias controle (água salina e clorexidina $0,12 \%$ ). O teste foi realizado em triplicata e os náuplios vivos foram contados após $24 \mathrm{~h}$. A concentração letal média (CL50) foi obtida e submetida a análise Probit através do

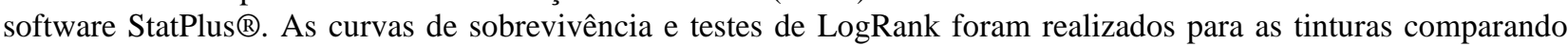
com os controles, através do software GraphPad® Prism. A CL50 ( $\mu \mathrm{g} / \mathrm{ml} \pm$ erro-padrão) para a malva foi de $276,08 \pm$ 41,12, e para a calêndula 268,95 $\pm 44,09$, sendo ambas as tinturas consideradas ativas. Verificou-se diferença estatística na curva de sobrevivência tanto para a malva quanto para a calêndula quando comparadas com solução salina $(\mathrm{p}<0,0001)$, indicando maior toxicidade das tinturas. Também houve diferença estatística quando as tinturas foram comparadas a clorexidina $(\mathrm{p}<0,0001)$, indicando maior toxicidade da clorexidina. Não houve diferença na taxa de sobrevivência dos náuplios, quando analisadas as tinturas de malva e calêndula $(\mathrm{p}=0,8089)$. Pôde-se concluir que as tinturas de malva $10 \%$ e calêndula $10 \%$ apresentaram toxicidade moderada frente a Artemia salina.
\end{abstract}

Palavras-chave: Malva; Calendula; Fitoterapia; Testes de toxicidade aguda.

\begin{abstract}
The objective was to evaluate the toxicity of tinctures of mallow 10\% (Malva sylvestris) and marigold $10 \%$ (Calendula officinalis), through the bioassay with Artemia salina. The nauplii were obtained and transferred to test tubes, which contained mallow and marigold tinctures in different concentrations $(25,50,75,150,250,500,750$ and $1000 \mu \mathrm{g} / \mathrm{ml}$ ), and the control substances (saline water and $0.12 \%$ chlorhexidine. The test was performed in triplicate and live nauplii were counted after $24 \mathrm{~h}$. The mean lethal concentration (LC50) was obtained and submitted to Probit analysis using the StatPlus ${ }^{\circledR}$ software. Survival curves and LogRank tests were performed for the tinctures compared
\end{abstract}


to controls, using the GraphPad® Prism software. The LC50 ( $\mu \mathrm{g} / \mathrm{ml} \pm$ standard deviation) for mallow was $276.08 \pm$ 41.12, and for marigold $268.95 \pm 44.09$, both tinctures being considered active. There was a statistical difference in the survival curve for both mallow and marigold when compared to saline solution $(\mathrm{p}<0.0001)$, indicating greater toxicity of the tinctures. There was also a statistical difference when the tinctures were compared to chlorhexidine $(\mathrm{p}<0.0001)$, indicating greater toxicity of chlorhexidine. There was no difference in the survival rate of nauplii, when the mallow and marigold tinctures were analyzed $(\mathrm{p}=0.8089)$. It was concluded that the tinctures of mallow $10 \%$ and marigold $10 \%$ presented moderate toxicity against Artemia salina.

Keywords: Malva; Calendula; Phytotherapy; Toxicity tests, acute.

\section{Resumen}

El objetivo fue evaluar la toxicidad de las tinturas de malva 10\% (Malva sylvestris) y caléndula $10 \%$ (Calendula officinalis), mediante el bioensayo con Artemia salina. Los nauplios se obtuvieron y transfirieron a tubos de ensayo, los cuales contenían tinturas de malva y caléndula en diferentes concentraciones $(25,50,75,150,250,500,750$ y $1000 \mu \mathrm{g} / \mathrm{ml}$ ), y las sustancias de control (agua salina y $0.12 \%$ clorhexidina. La prueba se realizó por triplicado y se contaron los nauplios vivos después de 24 h. La concentración letal media (CL50) se obtuvo y se sometió a análisis Probit utilizando el software StatPlus ${ }^{\circledR}$. Se realizaron curvas de supervivencia y pruebas de LogRank para los tintes en comparación con los controles, utilizando el software GraphPad® Prism. La CL50 ( $\mu \mathrm{g} / \mathrm{ml} \pm$ desviación estándar) para malva fue 276,08 $\pm 41,12$ y para caléndula 268,95 $\pm 44,09$, considerándose ambos colorantes activos. Hubo una diferencia estadística en la curva de supervivencia tanto para la malva como para la caléndula en comparación con la solución salina ( $\mathrm{p}<0,0001)$, lo que indica una mayor toxicidad de las tinturas. También hubo una diferencia estadística cuando se compararon los tintes con la clorhexidina ( $\mathrm{p}<0,0001$ ), lo que indica una mayor toxicidad de la clorhexidina. No hubo diferencia en la tasa de supervivencia de nauplios, cuando se analizaron las tinturas de malva y caléndula $(\mathrm{p}=0,8089)$. Se concluyó que las tinturas de malva $10 \%$ y caléndula $10 \%$ presentaron toxicidad moderada frente a Artemia salina.

Palabras clave: Malva; Calendula; Fitoterapia; Pruebas de toxicidad aguda.

\section{Introdução}

A natureza é fonte de uma ampla variedade de plantas reconhecidas pelos seus valores medicinais, o que levou à descoberta de vários ativos químicos farmacológicos, utilizados em diferentes formas farmacêuticas (Benso et al., 2015). As plantas medicinais são empregadas na medicina popular, sendo um recurso importante no processo de prevenção e tratamento de doenças (Barroso et al., 2021; Hocayen et al., 2012).

As plantas medicinais têm sido cada vez mais estudadas, por apresentarem eficácia comprovada, menor resistência microbiana, menor toxicidade a curto e longo prazo, gerar poucos efeitos adversos e pelo baixo custo (Bohneberger et al., 2019; Razavi et al., 2011). Uma das áreas que se observa uma crescente utilização dos fitoterápicos é na Odontologia (Bohneberger et al., 2019; Domingues et al., 2021), pois muitas plantas apresentam atividades farmacológicas, antiinflamatória, antifúngica, analgésica, antibiótica, antivirais, antibiofilme e de cicatrização de feridas, ações importantes para o tratamento de doenças bucais (Araújo et al., 2021; Faria et al., 2011; Mahyari et al., 2016; Simões \& Oliveira Filho, 2021; Sousa et al., 2021; Tanideh et al., 2020). Dentre as plantas medicinais pode-se destacar a malva (Malva sylvestris) e a calêndula (Calendula officinalis), devido a eficácia, por serem de fácil aquisição e muito utilizadas (Freires \& Rosalen, 2016; Oliveira, 2021; Vinagre et al., 2011).

A malva pertence à família Malvaceae, é uma planta nativa da Europa, África e Ásia, conhecida popularmente como malva, malva-alta, malva-silvestre, malva-verde, malva-selvagem (Bohneberger et al., 2019). Suas folhas, flores e raízes são muito utilizadas, devido as propriedades anti-inflamatórias, antisséptica, antioxidante, anticâncer, antiúlcera calmantes, emolientes e antimicrobianas. Sendo indicada na Odontologia, para tratamento dos processos inflamatórios da boca, na doença periodontal e de feridas em mucosa, além disso, possui ação frente às bactérias formadoras do biofilme dental e ação anti-cárie comparável com a clorexidina, com menos efeito citotóxico (Benso et al., 2015; Bohneberger et al., 2019; Braga, Pires, \& Magalhães, 2018; Kovalik et al., 2014; Razavi et al., 2011; Simões \& Oliveira Filho, 2021; Sousa et al., 2021). Seus principais constituintes químicos são mucilagem, taninos, naftaquinonas, óleos essenciais, flavonoides, eugenol, fitol e ácidos 
cumarínico, clorogênico, cafeico, oleico, palmítico e esteárico (Bohneberger et al., 2019; Kovalik et al., 2014; Oliveira, 2021; Razavi et al., 2011; Sousa et al., 2021). Na Odontologia a malva é muito utilizada como colutórios bucais (Kovalik et al., 2014).

A calêndula é uma planta herbácea pertencente à família Asteraceae, é uma planta nativa da região do Mediterrâneo, norte da África e Ásia, e amplamente disseminada no Brasil. É conhecida popularmente como calêndula, malmequer, maravilha dos jardins, margarida dourada, sendo utilizadas para o preparo de fitoterápicos e também com fins ornamentais. Utiliza-se as flores e folhas, sendo os principais constituintes químicos as saponinas triterpênicas, carotenoides, flavonoides, polissacarídeos, hidroxicumarinas e sesquiterpenos (Bohneberger et al., 2019; Gazim et al., 2007; Uribe-Fentanes et al., 2018). A calêndula é utilizada na Odontologia para tratamento de estomatites aftosas, como irrigantes no tratamento endodôntico e após exodontias, pela ação antimicrobiana, anti-inflamatória, analgésica, antiedematosa, por promover hemostasia, antirreabsortivos e auxiliar na cicatrização, devido ao efeito colagenogênico e no metabolismo ósseo, preservando o osso alveolar (Alexandre et al., 2018; Bohneberger et al., 2019; Faria et al., 2011; Gupta et al, 2020; Mahyari et al., 2016; Nuñez et al., 2021; Parente et al., 2009; Uribe-Fentanes et al., 2018).

Apesar de serem produtos naturais, eles podem apresentam toxicidade e, por esse motivo, necessitam serem testados, para que sejam utilizadas com segurança. Um dos testes frequentemente utilizados, é o da toxicidade aguda com Artemia salina, como avaliação preliminar de toxicidade (Hocayen et al., 2012; Martins et al., 2021; Meyer et al., 1982). A Artemia salina é um microcrustáceo, utilizado para esse tipo de teste por serem sensíveis às variações ambientais, pela facilidade da aquisição dos cistos de Artemia salina e por permanecer viável no estado seco durante anos, apresentar baixo custo, fornece uma grande quantidade de náuplios e por ser uma alternativa a outros ensaios que utilizam animais (Freires et al., 2016; Meyer et al., 1982; Pelka et al., 2000). Através desse ensaio é obtida a concentração letal média (CL50), que é à concentração de um produto químico no ar ou na água que leva à morte de $50 \%$ de uma população em teste num tempo pré-estabelecido, sendo consideradas tóxicas as substâncias que apresentarem valores de CL50 abaixo de $1000 \mu$ g/ml (Bueno \& Piovezan, 2015; Martins et al., 2021; Meyer et al., 1982; Minho, Gaspar, \& Domingues, 2017; Queiroz \& Lima, s/d).

Diante do exposto, o objetivo desse estudo foi avaliar a toxicidade das tinturas de malva 10\% e calêndula $10 \%$ através do bioensaio com Artemia salina.

\section{Metodologia}

Trata-se de uma pesquisa laboratorial, de natureza quantitativa (Pereira et al., 2018), onde foi testado a toxicidade de tinturas de malva e calêndula, as quais foram obtidas de acordo com as diretrizes da Farmacopeia Brasileira (Agência Nacional de Vigilância Sanitária, 2021), na Farmácia Fleming Manipulação (Ponta Grossa, Paraná, Brasil). Utilizou-se o bioensaio com Artemia salina, de acordo com a técnica descrita por Meyer et al. (1982).

Os cistos secos de Artemia salina (Maramarpet, Arraial do Cabo, Rio de Janeiro, Brasil) foram obtidos em loja de aquarismo. Os cistos (3 g) foram incubados em um aquário de vidro com água salina artificial (30 g de sal marinho para 1,5 1 de água destilada) à temperatura de $28^{\circ} \mathrm{C}$ e com aeração constante, simulando o ambiente natural da Artemia salina (água salina rasa). Foi mantida iluminação constante $(20 \mathrm{~W})$, e o pH mantido entre 8 e 9, controlado com bicarbonato de sódio e verificado através de tira universal de pH. As larvas não receberam alimentação durante o período do estudo (Martins et al., 2021; Meyer et al., 1982).

Após 30 horas (período de eclosão dos cistos), os náuplios de Artemia salina foram coletados com pipetas plásticas de $3 \mathrm{ml}$ e 10 náuplios foram transferidos para cada tubo de ensaio (tinturas de malva e calêndula nas diferentes concentrações, controle positivo e negativo). $\mathrm{O}$ teste foi realizado em triplicata. 
Foram testadas as seguintes concentrações das tinturas de malva e calêndula a 10\%: $1000 \mu \mathrm{g} / \mathrm{ml}, 750 \mu \mathrm{g} / \mathrm{ml}, 500$ $\mu \mathrm{g} / \mathrm{ml}, 250 \mu \mathrm{g} / \mathrm{ml}, 150 \mu \mathrm{g} / \mathrm{ml}, 100 \mu \mathrm{g} / \mathrm{ml}, 50 \mu \mathrm{g} / \mathrm{ml}$ e $25 \mu \mathrm{g} / \mathrm{ml}$. Utilizou-se solução salina como controle negativo e digluconato de clorexidina $0,12 \%$ (Clorexidin, Dentalclean, Londrina, Paraná) como controle positivo. O controle negativo foi utilizado para se ter certeza de que a mortalidade observada nos náuplios era resultante da toxicidade aos compostos e não devido à falta de alimentação. E a clorexidina como controle positivo, devido ao fato da malva e da calêndula serem ativos interessantes para serem adicionados em enxaguatórios, tornando-se possível verificar a toxicidade das tinturas frente aos náuplios e comparar com uma substância muito utilizada como enxaguatório bucal.

Os náuplios vivos foram contados após 24h. Foram considerados vivos todos aqueles que apresentassem qualquer tipo de movimento quando observado próximo a uma fonte luminosa, durante 10 segundos. Cada tubo de ensaio foi avaliado por duas pessoas. Quando havia divergência nas contagens, o procedimento era repetido pelos operadores.

Para avaliar CL50, os dados foram submetidos a análise de Probit através do software StatPlus® (AnalystSoft, USA), após $24 \mathrm{~h}$ de exposição as substâncias testadas. As tinturas foram consideradas ativas quando os valores foram menores que $1000 \mu \mathrm{g} / \mathrm{ml}$. Curvas de sobrevivência e testes de LogRank foram realizados para os extratos comparando com os controles negativo e positivo, através do software GraphPad® Prism (GraphPad Software, San Diego, California, EUA).

\section{Resultados}

Os valores de CL50 \pm erro-padrão e os respectivos intervalos de confiança, de todas as preparações das plantas medicinais estão apresentados na Tabela 1 . O nível de intervalo de confiança foi de $1 \%$.

Tabela 1 - Dados de toxicologia obtidos para as plantas medicinais no bioensaio de Artemia salina.

\begin{tabular}{lcccc}
\hline Planta Medicinal & $\begin{array}{c}\text { CL50 } \\
(\boldsymbol{\mu g} / \mathbf{m l})\end{array}$ & Erro-padrão & $\begin{array}{c}\text { Limite inferior } \\
(\boldsymbol{\mu g} / \mathbf{m l})\end{array}$ & $\begin{array}{c}\text { Limite superior } \\
(\boldsymbol{\mu g} / \mathbf{m l})\end{array}$ \\
\hline Malva & 276,08 & 41,12 & 169,18 & 382,98 \\
Calêndula & 268,95 & 44,09 & 155,33 & 384,57 \\
\hline
\end{tabular}

Fonte: Autores.

As concentrações de $1000 \mu \mathrm{g} / \mathrm{ml}, 750 \mu \mathrm{g} / \mathrm{ml}, 500 \mu \mathrm{g} / \mathrm{ml}, 250 \mu \mathrm{g} / \mathrm{ml}, 150 \mu \mathrm{g} / \mathrm{ml}, 100 \mu \mathrm{g} / \mathrm{ml}, 50 \mu \mathrm{g} / \mathrm{ml}$ e $25 \mu \mathrm{g} / \mathrm{ml}$ foram utilizadas para o cálculo da CL 50, em que é estabelecida a Concentração Letal para 50\% da Artemia salina. Foram realizadas as curvas de sobrevivência e testes de LogRank para todas as concentrações testadas para se chegar ao resultado da CL50. Foi representado o percentual de sobrevivência na concentração de $250 \mu \mathrm{g} / \mathrm{ml}$ por ser a concentração que mais se aproximou do valor obtido da CL50 para a malva $276 \mu \mathrm{g} / \mathrm{ml}$ e para a calêndula $268 \mu \mathrm{g} / \mathrm{ml}$.

As curvas de sobrevivência dos náuplios testadas com as tinturas de malva $10 \%$ e calêndula $10 \%$, nas concentrações de $250 \mu \mathrm{g} / \mathrm{ml}$, estão representadas nas Figuras 1 e 2 respectivamente, contra os respectivos grupos controle negativo e positivo, acompanhados dos valores calculados na análise estatística para o teste de LogRank, durante $24 \mathrm{~h}$. 
Figura 1 - Porcentagem de sobrevivência dos náuplios de Artemia salina, malva versus solução salina e malva versus digluconato de clorexidina.
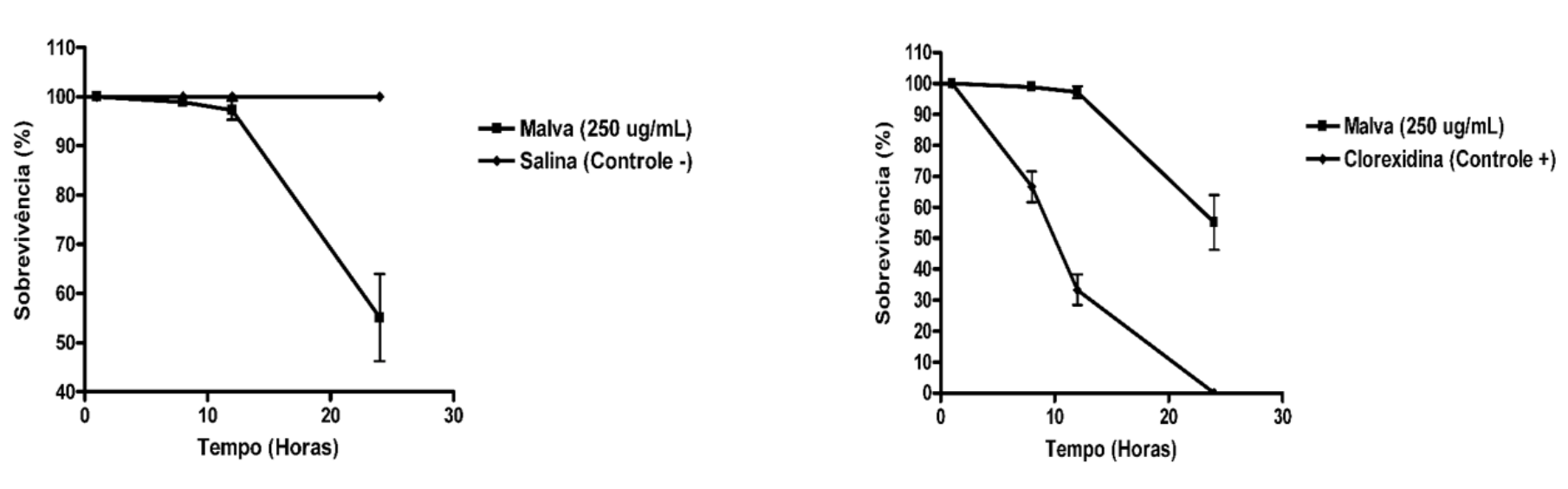

Fonte: Autores.

Verificou-se diferença estatística na curva de sobrevivência para a malva ( $\mathrm{p}<0,0001)$ quando comparada com solução salina. Quando a malva foi comparada com o digluconato de clorexidina, observou-se diferença significante (p < 0,0001), indicando que o digluconato de clorexidina é mais tóxico que a tintura de malva.

Figura 2 - Porcentagem de sobrevivência dos náuplios de Artemia salina, calêndula versus solução salina e calêndula versus digluconato de clorexidina.
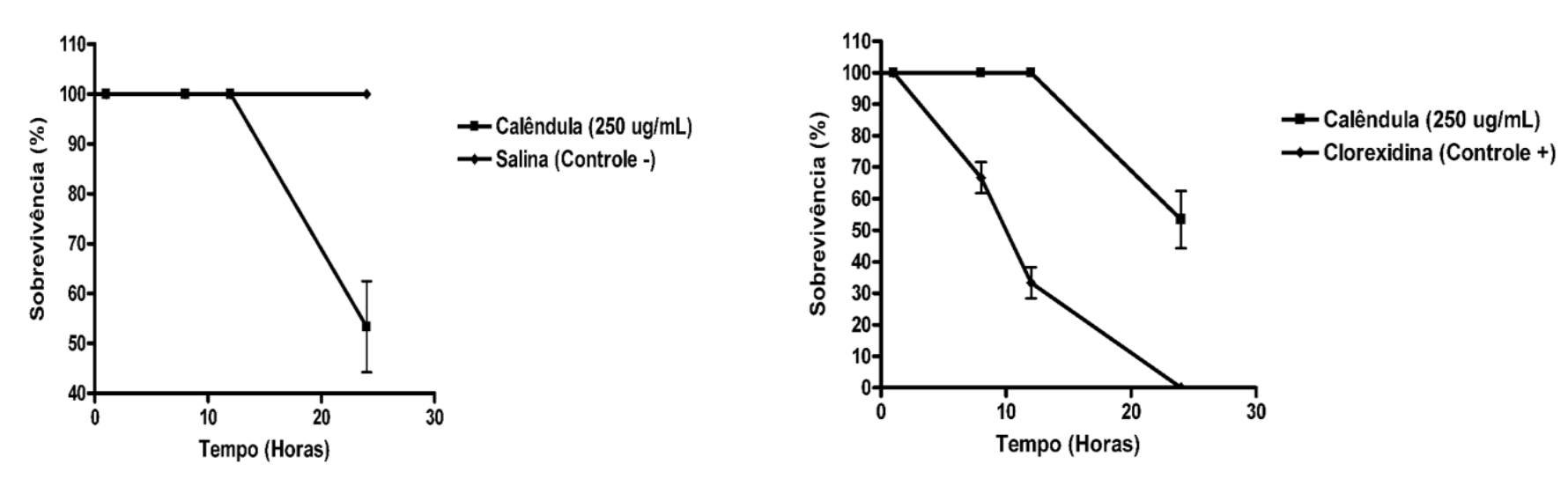

Fonte: Autores.

Verificou-se diferença estatística na curva de sobrevivência, quando a tintura de calêndula foi comparada com solução salina ( $\mathrm{p}<0,0001$ ), demonstrando maior toxicidade da calêndula. Quando a calêndula foi comparada com o digluconato de clorexidina, observou-se diferença significante $(\mathrm{p}<0,0001)$, indicando que o digluconato de clorexidina apresenta maior toxicidade que a calêndula.

Não foi observada diferença na taxa de sobrevivência da Artemia salina, quando analisadas as tinturas de malva e calêndula ( $\mathrm{p}=0,8089)$, segundo o teste de Logrank, como demonstrado na Figura 3. 
Figura 3 - Porcentagem de sobrevivência dos náuplios de Artemia salina frente a amostras de malva versus calêndula.

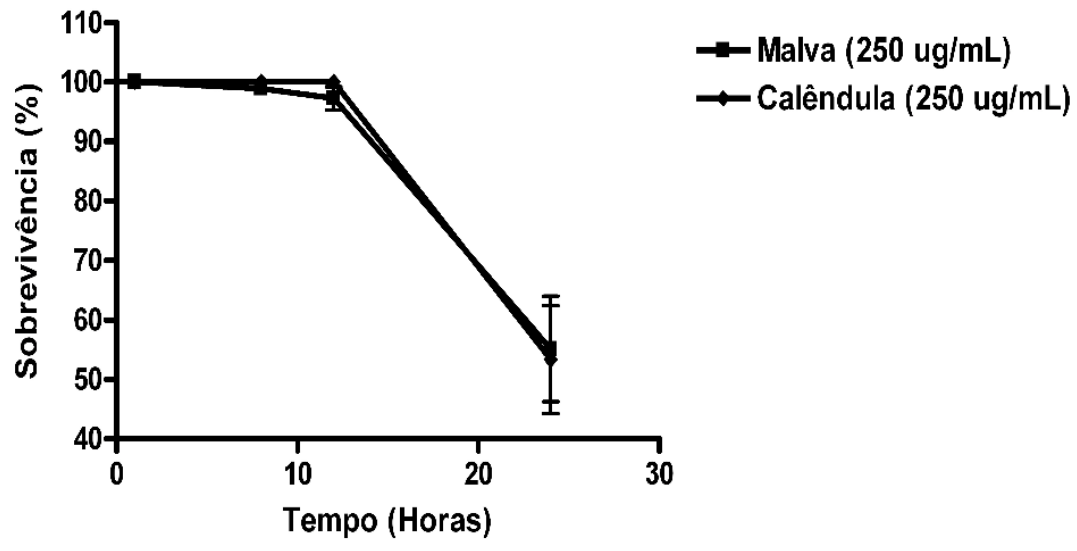

Fonte: Autores.

\section{Discussão}

Optou-se por utilizar as tinturas a 10\%, porque se aproxima da realidade de pacientes que possuem doenças bucais e utilizam como tratamento, a malva e a calêndula na forma de chá. Além disso, facilita o trabalho de diluição das diferentes soluções aquosas a serem testadas a partir do preparo das tinturas a $10 \%$.

Os resultados da CL50 (malva $=276,08 \mu \mathrm{g} / \mathrm{ml}$ e calêndula $=268,95 \mu \mathrm{g} / \mathrm{ml}$ ) obtidos através do bioensaio com Artemia salina, indicaram toxicidade moderada para as tinturas avaliadas, pois apresentaram valores para CL50 entre 100 a $500 \mu \mathrm{g} / \mathrm{ml}$ (Amarante et al., 2011). Tanto a tintura de malva 10\%, quanto a de calêndula 10\%, apresentaram maior toxicidade quando comparadas à solução salina, essa diferença era esperada, pois a solução salina é o meio em que a Artemia salina vive. Porém, quando comparadas ao digluconato de clorexidina, substância contida frequentemente em produtos odontológicos, as duas tinturas apresentaram menor toxicidade.

Em um estudo que utilizou a mesma metodologia e avaliou a toxicidade da tintura de romã, o resultado da CL50 não diferiu dos resultados do presente estudo, sendo também considerada de moderada toxicidade (Martins et al., 2021). As tinturas de malva e calêndula apresentaram menor toxicidade quando comparadas ao óleo essencial de copaíba $(80,77 \mu \mathrm{g} / \mathrm{ml})$ e óleo essencial de gerânio $(55,04 \mu \mathrm{g} / \mathrm{ml})$, contudo apresentaram maior toxicidade quando comparada ao óleo essencial de capimlimão $(900,7 \mu \mathrm{g} / \mathrm{ml})$ e a tintura de aroeira $(607,87 \mu \mathrm{g} / \mathrm{ml})$ (Lima et al., 2021; Martins et al., 2021).

Ao consultarmos a literatura observamos que os resultados de toxicidade dos fitoterápicos em ensaios com Artemia salina apresentam bastante variação, o que pode ser justificado por serem compostos por diferentes constituintes químicos, apesar de muitas vezes apresentarem atividades farmacológicas semelhantes. Esses aspectos devem ser considerados na escolha das plantas medicinais.

Agentes naturais foram adicionados em enxaguatórios bucais comerciais, visando a redução de efeitos colaterais em comparação com os agentes convencionais. Braga et al. (2018) compararam a efetividade da malva com diferentes enxaguatórios bucais e verificaram que a malva possui ação anticárie comparável aos demais enxaguatórios bucais. Portanto, pode ser uma interessante alternativa à clorexidina, uma vez que é um agente natural com baixo potencial citotóxico (Benso et al., 2015). O que corrobora com os resultados do presente estudo, pois observamos que as tinturas de malva e calêndula apresentam menor toxicidade quando comparada à clorexidina $0,12 \%$.

Resultados semelhantes pôde ser verificado na pesquisa clínica de Vinagre et al. (2011) que compararam o desempenho de um enxaguatório bucal contendo tintura de calêndula, com um colutório com clorexidina a $0,12 \%$, na melhoria 
da saúde periodontal. Concluíram que o enxaguatório fitoterápico pode ser sugerido, pois contribui com a saúde periodontal, apresentando o mesmo desempenho do colutório com clorexidina. Além da desvantagem já apresentada em relação a maior toxicidade da clorexidina, quando utilizada por um período de tempo mais longo pode causar manchamento dental, alteração do paladar e lesões descamativas na mucosa bucal, o que pode ser evitado ao utilizar enxaguatórios contendo fitoterápicos (Araújo et al., 2021; Pegoraro et al., 2015).

O bioensaio realizado com Artemia salina, mostrou-se bastante viável pelo custo acessível, o material ser de fácil aquisição, não precisar de equipamentos especiais e ser seguro. Apesar da técnica fornecer resultados confiáveis, ser possível controlar as variáveis, trata-se de uma avaliação preliminar de toxicidade, necessitando de avaliações com outras metodologias para utilização segura em humanos. Além disso, deve-se comparar tinturas de diferentes fornecedores e avaliar a toxicidade das tinturas de malva e calêndula quando presentes em formulações de enxaguatórios.

\section{Considerações Finais}

Pôde-se concluir que as tinturas de malva 10\% e calêndula 10\% apresentaram toxicidade moderada frente a Artemia salina.

Sugere-se a realização de pesquisas que avalie a toxicidade e possíveis efeitos adversos de diferentes formulações contendo malva e calêndula, para que possam ser utilizadas clinicamente com segurança, visto que as propriedades das plantas medicinais testadas permitem diferentes aplicações clínicas.

\section{Referências}

Agência Nacional de Vigilância Sanitária. (2021). Formulário de Fitoterápicos da Farmacopéia Brasileira. https://www.gov.br/anvisa/ptbr/assuntos/farmacopeia/formulario-fitoterapico/arquivos/2021-fffb2-final-c-capa2.pdf

Alexandre, J. T. M., Sousa, L. H. T., Lisboa, M. R. P., Furlaneto, F. A., do Val, D. R., Marques, M., \& Brito, G. A. C. (2018). Anti-inflammatory and antiresorptive effects of Calendula officinalis on inflammatory bone loss in rats. J Clinical oral investigations, 22(6), 2175-2185.

Amarante, C. B. d., Müller, A. H., Póvoa, M. M., \& Dolabela, M. F. (2011). Estudo fitoquímico biomonitorado pelos ensaios de toxicidade frente à Artemia salina e de atividade antiplasmódica do caule de aninga (Montrichardia linifera). J Acta Amazonica, 41, 431-434.

Araújo, J., Dobrovolski, M., Kozlowski Júnior, V. A., \& Rezende, M. (2021). Uso de solução de Plantago major para o controle do biofilme dental: relato de caso. J Research, Society Development, 10(1), e29610111491-e29610111491.

Barroso, L. K. D., Leal, N. d. A. C., Fontineles, C. F. F., Lopes, I. R. C., do Monte Rodrigues, J. A., Farias, M. S., do Vale Silva, A. (2021). Educação Permante em Saúde: uma estratégia para capacitação de profissionais da Estratégia de Saúde da Família. J Brazilian Journal of Development, 7(4), 3735837365 .

Benso, B., Rosalen, P. L., Alencar, S. M., \& Murata, R. M. (2015). Malva sylvestris Inhibits Inflammatory Response in Oral Human Cells. An In Vitro Infection Model. PLoS One, 10(10), e0140331. 10.1371/journal.pone.0140331

Bohneberger, G., Machado, M. A., Debiasi, M. M., Dirschnabel, A. J., \& de Oliveira Ramos, G. (2019). Fitoterápicos na odontologia, quando podemos utilizálos? J Brazilian Journal of Health Review, 2(4), 3504-3517.

Braga, A. S., Pires, J. G., \& Magalhães, A. C. (2018). Effect of a mouthrinse containing Malva sylvestris on the viability and activity of microcosm biofilm and on enamel demineralization compared to known antimicrobials mouthrinses. J Biofouling, 34(3), 252-261.

Bueno, A. C., \& Piovezan, M. (2015). Bioensaio toxicológico utilizando Artemia salina: fatores envolvidos em sua eficácia. J Instituto Federal de Santa Catarina.

Domingues, J. d. J., Oliveira, L. T. A., de Assis Costa, M. D. M., Silva, L. d. A. M., Nascimento, F., \& Dietrich, L. (2021). Uso de fitoterápicos e demais componentes vegetais e minerais na fabricação de produtos odontológicos naturais: Revisão de literatura. J Research, Society Development. 10(3), e57610313678-e57610313678.

Faria, R. L., Cardoso, L. M., Akisue, G., Pereira, C. A., Junqueira, J. C., Jorge, A. O., \& Santos Junior, P. V. (2011). Antimicrobial activity of Calendula officinalis, Camellia sinensis and chlorhexidine against the adherence of microorganisms to sutures after extraction of unerupted third molars. $J$ Appl Oral Sci, 19(5), 476-482. 10.1590/s1678-77572011000500007

Freires, I. A., \& Rosalen, P. L. (2016). How Natural Product Research has Contributed to Oral Care Product Development? A Critical View. Pharm Res, 33(6), 1311-1317. 10.1007/s11095-016-1905-5 
Research, Society and Development, v. 11, n. 3, e20511326255, 2022

Freires, I. A., Sardi, J. C., de Castro, R. D., \& Rosalen, P. L. (2016). Alternative Animal and Non-Animal Models for Drug Discovery and Development: Bonus or Burden? Pharm Res, 34(4), 681-686. 10.1007/s11095-016-2069-z

Gazim, Z. C., Ferreira, G. A., Rezende, C. M., Nakamura, C. V., Dias Filho, B. P., \& Cortez, D. A. G. (2007). Identificação dos constituintes químicos da fração volátil da Calendula officinalis produzida no Paraná. J Horticultura Brasileira, 25, 118-121.

Gupta, D., Kamat, S., Hugar, S., Nanjannawar, G., \& Kulkarni, R. (2020). A comparative evaluation of the antibacterial efficacy of Thymus vulgaris, Salvadora persica, Acacia nilotica, Calendula arvensis, and 5\% sodium hypochlorite against Enterococcus faecalis: An in-vitro study. J Conserv Dent, 23(1), 97-101. 10.4103/JCD.JCD_48_20

Hocayen, P. d. A. S., Campos, L. A., Pochapski, M. T., \& Malfatti, C. R. M. (2012). Avaliação da Toxicidade do extrato bruto metanólico de Baccharis dracunculifolia por meio do bioensaio com Artemia salina. J Insula Revista de Botânica (41), 23-31.

Kovalik, A. C., Bisetto, P., Pochapski, M. T., Campagnoli, E. B., Pilatti, G. L., \& Santos, F. A. (2014). Effects of an orabase formulation with ethanolic extract of Malva sylvestris L. in oral wound healing in rats. J Med Food, 17(5), 618-624. 10.1089/jmf.2013.0001

Lima, L. R., Andrade, F. K., Alves, D. R., de Morais, S. M., \& Vieira, R. S. (2021). Anti-acetylcholinesterase and toxicity against Artemia salina of chitosan microparticles loaded with essential oils of Cymbopogon flexuosus, Pelargonium x ssp and Copaifera officinalis. Int J Biol Macromol, 167, 1361-1370. 10.1016/j.ijbiomac.2020.11.090

Mahyari, S., Mahyari, B., Emami, S. A., Malaekeh-Nikouei, B., Jahanbakhsh, S. P., Sahebkar, A., \& Mohammadpour, A. H. (2016). Evaluation of the efficacy of a polyherbal mouthwash containing Zingiber officinale, Rosmarinus officinalis and Calendula officinalis extracts in patients with gingivitis: A randomized double-blind placebo-controlled trial. Complement Ther Clin Pract, 22, 93-98. 10.1016/j.ctcp.2015.12.001

Martins, A. C. R., da Costa, J. K. N., Herbert, A., Farias, F. R. S., Rezende, M., Junior, V. A. K., \& de Geus, J. L. (2021). Avaliação da toxicidade das tinturas de aroeira e de romã através do bioensaio com Artemia salina. J Research, Society Development, 10(3), e52010313751-e52010313751.

Meyer, B. N., Ferrigni, N. R., Putnam, J. E., Jacobsen, L. B., Nichols, D. E., \& McLaughlin, J. L. (1982). Brine shrimp: a convenient general bioassay for active plant constituents. Planta Med, 45(5), 31-34. 10.1055/s-2007-971236

Minho, A., Gaspar, E., \& Domingues, R. (2017). Guia prático para determinação de curva dose-resposta e concentração letal em bioensaios com extratos vegetais. J Embrapa Pecuária Sul-Comunicado Técnico.

Nuñez, A. I. B., Moreno-Montoya, A., Tejeda-Bertot, M. M., Griñán-Semanat, D. Y., \& Landazuri-Llago, S. (2021). Aplicación de una jalea de caléndula al $1 \%$ en pacientes con alveolitis. J Acta Odontológica Colombiana, 11(2).

Oliveira, J. M. (2021). Constituintes químicos e atividades biológicas de óleos essenciais das espécies Malva sylvestris e Pelargonium graveolens: uma revisão. J Revista Uningá Review, 36, eURJ3728-eURJ3728.

Parente, L. M. L., Silva, M., Lino-Júnior, R. d. S., Paula, J., Trevenzol, L., Zatta, D., \& Paulo, N. (2009). Efeito cicatrizante e atividade antibacteriana da Calendula officinalis L. cultivada no Brasil. J Revista Brasileira de Plantas Medicinais, 11, 383-391.

Pegoraro, J., Silvestri, L., Cara, G., Stefenon, L., \& Mozzini, C. B. J. J. o. O. I. (2015). Efeitos adversos do gluconato de clorexidina à 0, 12\%. $3(1)$, $33-37$.

Pelka, M., Danzl, C., Distler, W., \& Petschelt, A. (2000). A new screening test for toxicity testing of dental materials. J Dent, 28(5), 341-345. 10.1016/s03005712(00)00007-5

Pereira, A., Shitsuka, D., Parreira, F., \& Shitsuka, R. (2018). Metodologia da pesquisa científica UFSM, 119.

Queiroz, S., \& Lima, L. Tratado De Toxicologia Ocupacional: Doenças Profissionais Ou Do Trbalho: biblioteca24horas.

Razavi, S. M., Zarrini, G., Molavi, G., \& Ghasemi, G. (2011). Bioactivity of malva sylvestris L., a medicinal plant from iran. Iran J Basic Med Sci, 14(6), 574579.

Simões, A. P. G., \& Oliveira Filho, A. A. d. (2021). Plantas medicinais no combate ao biofilme dental: revisão da literatura. J Archives of Health Investigation, 10(3), 385-391.

Sousa, T. J. D., Araujo, L. K. O., Lima Filho, A. C. M., Gonçalves, C. A., Freitas, M. S., Ribeiro, M. R. G., \& Silva, C. S. (2021). O uso de plantas medicinais em infecções bucais: uma alternativa eficaz. J Revista Eletrônica Acervo Saúde, 13(4), e6880-e6880.

Tanideh, N., Ghafari, V., Ebrahimi, R., Habibagahi, R., Koohi-Hosseinabadi, O., \& Iraji, A. (2020). Effects of Calendula Officinalis and Hypericum Perforatum on Antioxidant, Anti-Inflammatory, and Histopathology Indices of Induced Periodontitis in Male Rats. J Dent (Shiraz), 21(4), 314-321. 10.30476/DENTJODS.2020.83660.1056

Uribe-Fentanes, L. K., Soriano-Padilla, F., Pérez-Frutos, J. R., \& Veras-Hernández, M. A. (2018). Acción del extracto de Calendula officinalis en la preservación ósea posterior a extracción. J Revista Médica del Instituto Mexicano del Seguro Social, 56(1), 98-105.

Vinagre, N. P. d. L., Farias, C. G., Araújo, R. J. G. d., Vieira, J. M. d. S., Silva Júnior, J. O. C., \& Corrêa, A. M. (2011). Efetividade clínica de um enxaguatório bucal fitoterápico com tintura padronizada de Calendula officinalis na manutenção da saúde periodontal. J Rev. odontol. UNESP, 30-35. 